\title{
Relación entre la comorbilidad psicopatológica y las variables de resultados en dependientes de cocaína tratados en comunidad terapéutica
}

\section{Relationship between psychopathological comorbidity and outcomes variables in-treatment cocaine dependent subject in therapeutic community}

\author{
Esperanza Vergara-Moragues ${ }^{*},{ }^{* *} ;$ Francisco \\ GONZÁLEZ-SAIZ ${ }^{\star \star *}$; OSCAR LOZANO-ROJAS ${ }^{\star \star \star *}$; \\ Fermín Fernández Calderón ${ }^{\star \star \star * * ; ~ A n t o n i o ~ V e r d e j o ~}$ \\ GARCía $^{\star * \star \star * *}$; PATRICIA Betanzos ESPINOSA**; IZASKUN \\ Bilbao Acedos ${ }^{\star \star * \star *}$; Miguel Pérez García ${ }^{* \star}$
}

\author{
* Departamento de Educación. Universidad Internacional de la Rioja (UNIR). \\ ** Centro de Investigación Mente, Cerebro y Comportamiento (CIMCYC). \\ Universidad de Granada. \\ *** Unidad de Salud Mental Comunitaria Villamartín. UGC Salud Mental Hospital \\ de Jerez (Cádiz). \\ ${ }_{* \star \star \star}$ Departamento de Psicología Clínica, Experimental y Social. Universidad de \\ Huelva. \\ ${ }_{* \star * \star \star *}$ Agencia de Servicio Sociales y Dependencia de Andalucía.

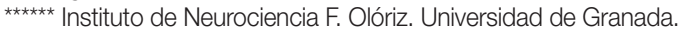 \\ Enviar correspondencia a: \\ Esperanza Vergara-Moragues. \\ Facultad de Psicología, Universidad de Granada, \\ Campus de Cartuja S/N, 18071 Granada, España. \\ Tel.: +34 958242948; fax: +34 958243749. \\ E-mail: esvermor@yahoo.es
}

recibido: Septiembre 2012 aceptado: Enero 2013

\section{Resumen}

El ingreso en comunidad terapéutica de personas con problemas de consumo de cocaina y comorbilidad psicopatológica ha ido aumentando en los últimos años y es importante conocer más sobre el éxito de estos tratamientos. El objetivo de este trabajo es estudiar el perfil psicopatológico de los pacientes dependientes de cocaína y su asociación con las variables de resultados. Se entrevistaron mediante la Psychiatric Research Interview for Substance and Mental Disorders (PRISM-IV) y la Conners' Adult ADHD Diagnostic Interview for DSM-IV (CAADID) a una muestral total de 218 pacientes que solicitaron tratamiento en alguna de las seis comunidades terapéuticas públicas de Andalucía. Los resultados exponen que el porcentaje de retención a los tres meses es del 68.3\% (149). De los cuales, el 43.5\% abandona el recurso con un alta terapéutica y el $52.8 \%$ con cambios clínicamente relevantes. Además, el 57.8\% presentan comorbilidad psicopatológica. Por último, existe un mayor éxito terapéutico (medidos por dias de estancias, tipo de altas y objetivos cumplido tras el tratamiento) entre las personas sin comorbilidad psicopatológica. Estos datos sugieren la necesidad de adaptar las comunidades terapéuticas en el tratamiento de personas dependientes de cocaina con comorbilidad psicopatológica y, de esta manera, mejorar el éxito terapéutico.

Palabras clave: Cocaina, comunidad terapéutica, tratamiento, psicopatología, trastornos por consumo de sustancias

\section{Abstract}

Over the last years, the therapeutic community treatment with people who have cocaine problems and psychopathological comorbidity has been increasing and it is important to know more about the success of these treatments. The aim of this paper is to study the psychopathological profile of cocaine-dependent patients and its association with outcome variables. It is interviewed by the Psychiatric Research Interview for Substance and Mental Disorders (PRISM-IV) and the Conners' Adult ADHD Diagnostic Interview for DSM-IV (CAADID) a total sample of 218 patients who sought treatment at any of the six public therapeutics community of Andalusia (Spain). The results show that the retention rate after three months of $68.3 \%$ (149), 43.5\% that leaves the resource with a therapeutic discharge and $52.8 \%$ with clinically significant changes. Besides, the $57.8 \%$ have psychopathological comorbidity. Finally, there is a higher treatment success (measured by days of stay, type of discharge and therapeutic community outcome clinical impression) among people without psychopathological comorbidity. These data show the need to adapt the therapeutic communities to treat people with comorbid psychopathology and, thus, improve therapeutic success. substance use disorder 
$\mathrm{E}$ consumo problemático de cocaína ha venido aumentando en los últimos años, siendo España uno de los países europeos con indicadores epidemiológicos y asistenciales más preocupantes (European Monitoring Centre for Drugs and Drugs Addiction, 2011). Junto a esto, uno de los factores que más preocupan a los clínicos es la comorbilidad psiquiátrica o patología dual. La coincidencia en el tiempo de un trastorno por consumo de sustancia y otro tipo de trastornos psicopatológico puede suponer por un lado, la complicación del diagnóstico y el tratamiento y, por el otro, un factor de mal pronóstico al asociarse a una peor respuesta al tratamiento (Vergara-Moragues, 2010). La prevalencia de psicopatología en población consumidora de cocaína es elevada, oscilando entre un 30-60 \% (Falck, Wang, Siegal y Carlson, 2004; Herrero, Domingo-Salvany, Brugal, Torrens e Itinere Investigators, 2008; Vergara-Moragues et al., 2012). El tratamiento de esta comorbilidad es complejo y se debe hacer desde un modelo de tratamiento integral fundamentado en el paradigma biopsicosocial, y teniendo como objetivo final lograr un cambio global en el estilo de vida de los pacientes (Bunt, Muehlbach y Moed, 2008).

La comunidad terapéutica (CT) para personas con problemas derivados del consumo de sustancias está indicada cuando los tratamientos ambulatorios son insuficientes para alcanzar los resultados deseables en una intervención de esta indole. En este ámbito, la prevalencia de comorbilidad psicopatológica es igualmente elevado (Mulder, Frampton, Peka, Hampton y Marsters, 2009; Sacks, McKendrick, Sacks y Cleland, 2010; Vergara-Moragues et al., 2012). Existen un número importante de estudios que se han dedicado a valorar la efectividad del tratamiento en CT. Como ejemplo, recientemente, Malivert, Fatséas, Denis, Langlois y Auriacombe (2012) realizaron una revisión sistemática donde seleccionaron 12 estudios de 321 encontrados $y$, concluyeron que, a pesar de que el porcentaje de recaída era importante tras el tratamiento, las personas que con intervención enT disminuían su consumo de sustancias. Otros autores señalan que en pacientes con trastornos por consumo de sustancias, particularmente aquellos que presentan comorbilidad psicopatológica, la CT es uno de los tratamientos que mejores cifras de remisión presen$\tan ($ De Leon, Sacks, Staines y McKendrick, 2000; Malivert et al., 2012; Mulder et al., 2009).

Pero, ¿a través de qué variables se puede evaluar los resultados de un tratamiento en este recurso asistencial? Todavía hoy, existe controversia sobre cómo valorar el éxito terapéutico en una CT. La mayoría de los autores están de acuerdo en considerar el tiempo de estancia como un indicador válido de resultados, siendo tres meses el tiempo medio estimulado para que el paciente pueda presentar una mejoría clínicamente relevante (Condelli y Hubbard, 1994; Greenfield, Burgdorf, Chen, Porowsk, Roberts y Herrell, 2004; Vergara-Moragues, 2010). Otros estudios longitudinales indican que algunas variables relacionadas con el paciente tienen un valor predictivo limitado sobre los resultados de tratamiento, al explicar un porcentaje de la varianza relativamente bajo de éstos (Condelli, 1994; Secades y Fernández, 2000). Por otro lado, otros autores, se limitan a estudiar un trastorno aislado (Mulder et al., 2009; Gilchrist, Langohr, Fonseca, Muga y Torrens, 2012;
Darke, Campbell y Popple, 2012; Tull y Gratz, 2012). Asimismo, no queda claro si la comorbilidad psicopatológica juega un valor predictivo en el éxito terapéutico en CT. Algunos trabajos le otorgan un papel neutro sobre la retención (Alterman, Rutherford, Cacciola, McKay y Woody, 1996; Malivert et al., 2012). Otros señalan que los pacientes con mayor gravedad de la sintomatología ansioso-depresiva permanecerían más tiempo en tratamiento (Agostini, Nunes, Stewart, Quitkin, 1991; Joe, Simpson y Broome, 1998). Finalmente, existen estudios que concluyen que tanto la gravedad de la psicopatología como la presencia de determinados diagnósticos psicopatológicos, conferirian un peor pronóstico respecto de la retención (Broome, Flynn y Simpson, 1999; Tull et al., 2012, Wise , Cuffe y Fischer, 2001). Estos últimos trabajos abren una interesante línea de investigación, ya que, aún se desconoce el valor predictivo especifico de cada trastorno psicopatológico sobre la retención en tratamiento.

Hasta nuestro conocimiento, ningún trabajo ha evaluado la relación entre la comorbilidad psicopatológica y las variables de resultados en CT con un instrumento como la Psychiatric Research Interview for Substances and Mental Diseases (PRISM) (Torrens, Serrano, Astals, Pérez-Domínguez y MartínSantos, 2004) específico para población con trastornos con consumo de sustancias.

Partiendo de las evidencias anteriores, el objetivo de este trabajo es estudiar la relación entre la comorbilidad psicopatológica y las variables de resultados como éxito terapéutico. La hipótesis a contrastar es que los pacientes dependientes de cocaína que presentan comorbilidad psicopatológica obtendrán un menor porcentaje de éxito terapéutico valorado en términos del tipo de alta y la impresión clínica tras el tratamiento.

\section{Método}

\section{Diseño}

Estudio descriptivo longitudinal.

\section{Participantes}

La población objetivo son pacientes con dependencia a cocaína que reciben tratamiento en CT. Como marco muestral se utilizaron las CTs públicas de Andalucía (Almonte, Cartaya, La Línea, Los Palacios, Mijas y Tarifa), y la muestra fue obtenida entre los pacientes que iniciaron tratamiento por consumo de cocaína en una de las CTs públicas de Andalucia entre Mayo de 2008 y Septiembre del 2010. Inicialmente estuvo previsto un muestreo aleatorio sistemático. Sin embargo, tras los primeros meses de estudio se optó por reclutar a todos los pacientes que reunían criterios de inclusión.

Los criterios de inclusión para participar en el estudio fueron: 1) dependencia de cocaína en los últimos 12 meses; 2) ser mayor de 18 años; 3) saber leer y escribir; 4) firmar el consentimiento 
informado. Los criterios de exclusión fueron: 1) deterioro cognitivo orgánico que dificulte la exploración psicopatológica; 2) tener pendiente el cumplimiento de una causa penal en los próximos 3 meses que impidiese el seguimiento en el centro de tratamiento 3) tener una abstinencia mayor de 45 días.

La muestra estuvo compuesta de 227 pacientes, si bien el análisis se ha realizados sobre 218 sujetos, ya que no se disponen de datos de 9 pacientes. La edad media de los pacientes participantes es de 34 años (DT=7.5), el 91.3\% de los sujetos eran hombres, más de la mitad (66.5\%) nunca se habian casado, tenían un nivel educativo bajo ( $84.9 \%$ con estudios primarios como máximo), estaban desempleados (68.3\%) y habian estado detenido alguna vez en su vida (59.2\%). El 78.4\% habían estado en tratamiento por consumo de sustancias antes de su ingreso en CT y el $43.6 \%$ en tratamiento psicológico o psiquiátrico (tabla 1).

Las seis CTs del estudio forman parte del sistema de salud público regional dedicado a los problemas de consumo de sustancias. Los protocolos asistenciales son iguales para todos los centros, y todas las CTs, tienen un programa de tratamiento multidisciplinar que incluye cuidados sanitarios (médicos y enfermería), educativos y psicológicos. Los programas de intervención fueron originalmente desarrollados y son periódicamente actualizados por un comité clínico formados por profesionales de los diferentes centros (Arenas, del Valle, López, Martín y Tirado, 2003). En éste se recoge que los pacientes que ingresan en CT vienen derivados de centros de tratamientos ambulatorios coordinados por la misma red de servicios de drogodependencias pública de Andalucía. Las razones principales de la derivación son: (i) incapacidad de mantener la abstinencia durante el tratamiento ambulatorio y/o (ii) decisión por parte de los profesionales clínicos de la necesidad de un tratamiento más intensivo y continuo. Las admisiones a las CT son siempre voluntarias, la asignación de los pacientes a los diferentes centros se maneja a través de un sistema de información computerizado en función de la disponibilidad de espacio (https://www.sipasda.info/).

\section{Instrumentos de Evaluación}

Psychiatric Research Interview for Substances and Mental Diseases (PRISM) (Torrens et al., 2004). La PRISM-IV es una entrevista clínica semiestructurada diseñada especialmente para evaluar la presencia de comorbilidad psicopatológica en los pacientes con trastorno por uso de sustancias. Evalúa, por un lado, los trastornos del Eje I y, por el otro, los dos trastornos psicopatológicos más prevalentes en esta población del Eje II (trastorno límite y trastorno antisocial de la personalidad). Además, a lo largo de la entrevista, se realiza un diagnóstico diferencial diferenciando si los trastornos son primarios/independientes o inducidos por el consumo de sustancias. El instrumento facilita los diagnósticos psiquiátricos actuales y los trastornos aparecidos a lo largo de la vida del paciente. Para el diagnóstico de los trastornos por uso de sustancias se empleó la sección de drogas de la PRISM. Con respecto a las características psicométrica de la entrevista, Hasin, Trautman, Miele, Samet, Smith y Endicott (1996) realizaron un primer análisis de fiabilidad test-retest con una muestra de 172 pacientes, observando unos parámetros de concordancia elevados para la mayoría de los diagnósticos obtenidos. En España, el grupo de Torrens, Serrano, Astals, Pérez-Dominguez y Martin-Santos (2004) realizaron un estudio de adaptación y validación de la versión española de la PRISM considerando como "patrón oro" los diagnósticos obtenidos a través del método Longitudinal Expert with All Data (LEAD). Este sistema se basa en la evaluación de los trastornos por parte de un experto a lo largo del tiempo, utilizando todos los datos disponibles para ello. Este trabajo se realizó con 105 pacientes con TCS y presentó una buena correlación entre los diagnósticos obtenidos mediante la PRISM y el sistema LEAD en TDM, psicosis inducida, TA y los dos trastornos de personalidad valorados (TAP y TLP) (Torrens et al., 2004).

Conners' Adult ADHD Diagnostic Interview for DSMIV (CAADID) (Conners et al., 1999). Este instrumento es una entrevista clínica semiestructurada que evalúa la presencia de cada uno de los criterios diagnósticos de TDAH según criterios DSM-IV-TR adaptados para adultos. En este estudio se ha empleado la versión española de este instrumento. Algunas de las propiedades psicométricas de la versión española son: sensibilidad del $98.86 \%$, especificidad del $67.68 \%$, valor predictivo positivo $90.77 \%$, valor predictivo negativo $94.87 \%$ y un índice de concordancia kappa entre pruebas (resultados obtenidos en la CAADID versus diagnóstico clínico) de 0.74 (SáezFrancàs et al., 2012). La CAADID está constituida por dos partes que se administran separadamente. La parte I es una entrevista que contiene preguntas sobre caracteristicas demográficas del paciente, explora el desarrollo de los síntomas de TDAH, los factores de riesgo asociados y preguntas sobre comorbilidad. La parte II está diseñada para identificar la presencia de los 18 criterios diagnósticos DSM-IV-TR en la edad adulta y en la infancia (de manera retrospectiva). Cada uno de los criterios por los que se pregunta se acompaña de ejemplos apropiados para la edad adulta con el propósito de aumentar la fiabilidad diagnóstica. Se ha seguido un procedimiento riguroso para valorar: 1) la presencia de los síntomas de desatención e hiperactividad/ impulsividad en distintos ámbitos de funcionamiento del sujeto; 2) el deterioro clínicamente significativo y 3) el diagnóstico diferencial de los síntomas presentes. Para este último aspecto, se trató de verificar, tal como exigen los criterios DSM-IV-TR, que los síntomas de TDAH presentes en cada paciente no estuviesen mejor explicados por otros diagnósticos psiquiátricos. Para ello se empleó la PRISM.

Medidas de resultados. Las medidas de resultados se registraron a través del Sistema de Información del Plan Andaluz sobre Drogas y Adicciones (siPASDA) (https://www.sipasda. info/). El siPASDA es un sistema de información computerizado que acumula información clínica y epidemiológica sobre los pacientes atendidos en los centros de atención especializada de Andalucia. Las medidas de resultados registradas fueron: el tipo de alta y la evaluación al alta del paciente o el tipo de abandono del recurso. Aunque la perspectiva clásica sobre las variables post-tratamiento considera estas dos últimas variables como variables relacionadas con el proceso de tratamiento, se pueden considerar también variables indirectas o variable subrogadas desde una perspectiva dentro del tratamiento. 
1. La retención en la CT fue definida como el número de días desde la admisión al tratamiento o tiempo de estancia, consistente con los criterios de Lewis y Ross (1994) (por ejemplo, tiempo de abandono).

2. La evaluación de la salida del paciente de CT se midió de dos formas:

a. Tipo de alta: Se han diferenciado tres tipos de altas. Alta terapéutica: cuando el paciente ha finalizado su proceso de tratamiento tras haber alcanzado significativamente los objetivos inicialmente planteados; alta por cumplimiento parcial de objetivos: se registra cuando se considera que el paciente ha tenido algún avance dentro del recurso, si bien, no ha cumplido con los objetivos planteados al comienzo del tratamiento; $y$, alta no terapéutica: engloba, a su vez, el "alta derivada por causas sociales, sanitarias o jurídicas", el "alta disciplinaria" (cuando el paciente incumple la normativa del centro), el "alta voluntaria" (cuando el paciente decide dar por terminado el tratamiento antes de cumplir cualquier objetivo planteado) y el "alta por exitus"(fallecimiento).

b. La impresión clínica al alta sobre la respuesta al tratamiento (ICA-RT). Sobre esta variable se han construido diferentes categorías: a) sin cambios: se asignó esta categoría a aquellas descripciones que ponen de manifiesto alguna de las siguientes condiciones: i) cuando el paciente mostró de manera permanente una actitud negativa durante el proceso terapéutico como, por ejemplo, contravenir las normas de convivencia, consumo o tráfico de sustancias en la CT, actitud desafiante o claramente agresiva; $y$, ii) cuando no fue posible realizar una primera valoración debido a su muy corta estancia en el recurso; b) cambios mínimos: se asignó a esta categoría aquellas descripciones que señalaban un mínimo avance (claramente insuficiente, desde el punto de vista clínico) en, al menos, una de las tres áreas de intervención (sanitaria, psicológica y socio-educativa), o bien, tras un cambio más relevante, se habia apreciado un retroceso en la evolución; y, c) cambios clínicamente relevantes: esta categoría corresponde a aquellos casos en los que se describe una mejoría mantenida y progresiva en una o más de las tres áreas de intervención. Es decir, se pone de manifiesto un cambio significativo durante todo el proceso asistencial, al alcanzarse claramente los objetivos terapéuticos planteados para esa o esas áreas.

El hecho de que las seis comunidades terapéuticas comparten un programa de tratamiento común y estandarizado hace que estas variables sean homogéneas en todos los centros.

\section{Procedimiento}

Todas las entrevistas fueron administradas por una única psicóloga clínica que tuvo un entrenamiento previo en la uti- lización de la PRISM-IV por parte del equipo de investigación que validó la versión española de este instrumento.

La entrevista inicial se realizó al ingreso en CT, durante la primera semana de estancia, y consistió en una breve entrevista de screening para evaluar los criterios de inclusión y exclusión del estudio, y valorar su incorporación al mismo. Posteriormente, los pacientes seleccionados fueron entrevistados entre los días 15 y 20 después de su ingreso en el recurso. Este intervalo fue elegido para evitar el efecto agudo de los síntomas de abstinencia o intoxicación que pueden interferir en la evaluación diagnóstica. Todos los pacientes que cumplian criterios de inclusión y no de exclusión del estudio que fueron invitados a participar aceptaron.

Los participantes no fueron compensados por su participación en el estudio.

\section{Análisis Estadisticos}

La caracterización de la muestra se ha realizado a través de análisis descriptivos, según el nivel de medida.

Para contrastar las diferentes hipótesis estadísticas se han aplicado ANOVA's (aplicando la prueba de Bonferroni para el estudio de las comparaciones post-hoc) y chi-cuadrado, en función de la naturaleza de las variables dependientes.

Los análisis se realizaron mediante paquete estadístico SPSS versión 15.0 (SPSS Inc., Chicago, IL).

\section{Resultados}

Como se observa en la tabla 1, de los 218 pacientes dependientes de cocaina tratados en CT, el $52 \%$ presentaban algún tipo de trastorno por consumo de heroína seguido del consumo por alcohol (41.7\%) y cannabis $(26.1 \%)$. Con respecto a la comorbilidad psicopatológica, la muestra presentaba una prevalencia del $57.8 \%$, dónde el trastorno antisocial de la personalidad (21.1\%) y los trastornos del estado de ánimo (20.2\%) fueron los más prevalentes, seguidos de los trastornos de ansiedad (15.6\%) y el TDAH (14.2\%).

De los 218 pacientes, el 56.5\% finaliza el tratamiento con un alta no terapéutica o con un alta por cumplimiento parcial de objetivos. El 43.5\% restante lo hace con un alta terapéutica. Por otro lado, como puede observarse en la tabla 2, la estancia media de estos pacientes fue de 139 días (DT=79); el $24.7 \%$ de la muestra finalizó el tratamiento sin cambios clínicos, el $22.5 \%$ con cambios mínimos, y el $52.8 \%$ con cambios clínicamente relevantes. El número de días de estancia es mayor en el grupo de pacientes que salieron de la CT con alta terapéutica (190 días), seguido de aquellos que tuvieron alta por cumplimiento parcial de objetivos (113 dias) y finalmente por los pacientes que salieron del centro con alta no terapéutica (71.6 días). No existieron diferencias en la retención entre los seis centros de tratamiento. 
Tabla 1. Variables sociodemográficas, trastornos por consumo de sustancias y comorbilidad psicopatológica

\begin{tabular}{|c|c|}
\hline$n=218$ & $\begin{array}{l}\text { Total } \\
\text { n (\%) }\end{array}$ \\
\hline \multicolumn{2}{|l|}{ VARIABLES SOCIODEMOGRÁFICA } \\
\hline Edad (media-desviación tipo) & $34(7.5)$ \\
\hline \multicolumn{2}{|l|}{ Sexo } \\
\hline Hombre & 199 (91.3) \\
\hline Mujer & $19(8.7)$ \\
\hline \multicolumn{2}{|l|}{ Estado Civil } \\
\hline Nunca casado & $145(66.5)$ \\
\hline Casado & $21(9.6)$ \\
\hline Divorciado/Separado/Viudo & $52(23.9)$ \\
\hline \multicolumn{2}{|l|}{ Nivel Educativo } \\
\hline$\leq$ Estudios Primarios & $185(84.9)$ \\
\hline$\geq$ Estudios Secundarios & $33(15.1)$ \\
\hline \multicolumn{2}{|l|}{ Empleo } \\
\hline Empleo & $45(20.6)$ \\
\hline Desempleo & $149(68.4)$ \\
\hline Jubilación/Incapacidad & $24(11)$ \\
\hline \multicolumn{2}{|l|}{ Alguna Detención } \\
\hline No & $89(40.8)$ \\
\hline Si & $129(59.2)$ \\
\hline \multicolumn{2}{|l|}{ Algún tratamiento por consumo de sustancias } \\
\hline No & $47(21.6)$ \\
\hline Si & $171(78.4)$ \\
\hline \multicolumn{2}{|l|}{ Algún tratamiento psicológico o psiquiátrico } \\
\hline No & $123(56.4)$ \\
\hline Si & $95(43.6)$ \\
\hline \multicolumn{2}{|l|}{ TRASTORNOS POR CONSUMO DE SUSTANCIAS EN EL ÚLTIMO AÑO } \\
\hline Abuso y/o dependencia de cocaina & $218(100)$ \\
\hline Abuso y/o dependencia de heroína & $114(52.3)$ \\
\hline Abuso y/o dependencia de alcohol & $91(41.7)$ \\
\hline Abuso y/o dependencia cannabis & $79(36.2)$ \\
\hline Abuso y/o dependencia sedantes/hipnóticos & $57(26.1)$ \\
\hline Abuso y/o dependencia alucinógenos & $3(1.4)$ \\
\hline Abuso y/o dependencia estimulantes & $7(3.2)$ \\
\hline Abuso y/o dependencia metadona+ & $16(7.3)$ \\
\hline Policonsumo (tres o más trastornos) & $19(8.7)$ \\
\hline \multicolumn{2}{|l|}{ COMORBILIDAD PSICOPATOLÓGICA EN EL ÚLTIMO AÑO } \\
\hline Algún trastornos psiquiátrico (Eje I y/o Eje II PRISM) & $126(57.8)$ \\
\hline Trastorno Eje I PRISM & $91(41.7)$ \\
\hline Trastorno del estado de ánimo & $44(20.2)$ \\
\hline Trastorno de Ansiedad & $34(15.6)$ \\
\hline Trastornos Psicótico & $37(17)$ \\
\hline Trastorno del EJE II PRISM & $64(29.5)$ \\
\hline Trastorno Antisocial de la Personalidad & $46(21.1)$ \\
\hline Trastorno Limite de la Personalidad & $30(13.8)$ \\
\hline Trastorno por Déficit de Atención e Hiperactividad CAADID & $31(14.2)$ \\
\hline
\end{tabular}

Nota: los números expresan medias y (desviaciones tipicas) para las variables cuantitativas y porcentajes para las variables cualitativas.. + Metadona no prescrita adquirida en el mercado negro.

Se detectaron diferencias estadísticamente significativas entre los días de estancia en CT y los tipos de alta, de tal forma que los días de estancia de los pacientes que finalizaron con alta terapéutica es superior a la observada en los otros dos tipos de altas. La comparación post-hoc mostró que existían diferencias estadísticamente significativas entre el grupo con alta terapéutica y los grupos con alta por cumplimiento parcial $(p<0.01)$ y no terapéutica $(p<0.01)$. No se observaron diferencias estadísticamente significativas entre estos dos últimos grupos $(p=0.092)$.
Por otro lado, esta misma tendencia se observó con la variable ICA-RT; es decir, un $91.6 \%$ de los pacientes que se marchaban del recurso con alta terapéutica obtienen cambios clínicamente relevantes frente a tan sólo un $28.8 \%$ con alta por cumplimiento parcial de objetivos $\left(\chi^{2}=140.875 ; p<0.01\right)$. Al contrario, la mayoría de personas con alta no terapéutica (62.5\%) no experimentan ningún tipo de cambio clínicamente relevante (sin cambios) durante el tiempo de estancia en el recurso.

Tabla 2. Comparación entre los tipos de alta y los días de estancia en CT vs la impresión clínica al alta sobre la respuesta al tratamiento (ICA-RT)

\begin{tabular}{|c|c|c|c|c|c|}
\hline \multicolumn{6}{|c|}{ TIPO DE ALTA } \\
\hline & TOTAL & $\begin{array}{c}\text { Alta no } \\
\text { terapéutica }\end{array}$ & $\begin{array}{c}\text { Alta por } \\
\text { cumplim- } \\
\text { iento parcial } \\
\text { de objetivos }\end{array}$ & $\begin{array}{c}\text { Alta tera- } \\
\text { péutica }\end{array}$ & $p$ \\
\hline $\begin{array}{r}\text { Días de estancia } \\
\text { en CT } \\
\text { Media (DT) }\end{array}$ & 139 (79) & $87(71.6)$ & $113(65)$ & $190(59.4)$ & $0.0001^{* *}$ \\
\hline ICA-RT & & & & & $0.0001^{* *}$ \\
\hline Sin cambios & 54 (24.8) & $40(62.5)$ & $11(18.6)$ & $3(3.2)$ & \\
\hline Cambios minimos & $49(22.5)$ & $13(20.3)$ & $31(52.5)$ & $5(5.3)$ & \\
\hline $\begin{array}{l}\text { Cambios clínica- } \\
\text { mente relevantes }\end{array}$ & $115(52.8)$ & $11(17.2)$ & $17(28.8)$ & 87 (91.6) & \\
\hline
\end{tabular}

Nota: los números expresan medias y (desviaciones típicas) para las variables cuantitativas y porcentajes para las variables cualitativas. ${ }^{*} p<0.05 ;{ }^{* *} p<0.01$

En esta línea de resultados, el porcentaje de retención al mes fue del $81.7 \%$ y a los tres meses del 68.3\%. El análisis de la ICA-RT y los días de estancia en $\mathrm{CT}$, mostró una relación estadisticamente significativa $(F=31.81 ; g . l .=2 ; p<0.01)$, indicando que en los pacientes con más de 90 dias de estancia en $\mathrm{CT}$, se observa un mayor porcentaje de cambios clínicamente relevantes $(71.1 \%)$ en comparación con los que permanecen entre uno (7.5\%) y tres meses (20.7\%) (ver tabla 3 ).

Tabla 3. Comparación entre los días de estancia en comunidad terapéutica y la impresión clínica al alta sobre la respuesta al tratamiento (ICA-RT)

\begin{tabular}{|c|c|c|c|c|c|}
\hline \multicolumn{5}{|c|}{ DÍAS ESTANCIA EN COMUNIDAD TERAPÉUTICA } & \\
\cline { 1 - 5 } $\begin{array}{c}\text { Impresión clínica } \\
\text { al alta sobre } \\
\text { la respuesta al } \\
\text { tratamiento } \\
\text { (ICA-RT) }\end{array}$ & TOTAL & $1-60$ días & $61-90$ días & $>90$ días & P \\
\hline Sin cambios & $54(24.8)$ & $28(70)$ & $11(37.9)$ & $15(10.1)$ & \multirow{2}{*}{$0.0001^{* *}$} \\
\hline Cambios minimos & $49(22.5)$ & $9(22.5)$ & $11(36.7)$ & $28(18.8)$ & \\
\hline $\begin{array}{c}\text { Cambios clinica- } \\
\text { mente relevantes }\end{array}$ & $115(52.8)$ & $3(7.5)$ & $6(20.7)$ & $106(71.1)$ & \\
\hline
\end{tabular}

${ }^{*} p<0.05 ;{ }^{* *} p<0.01$

La relación entre la presencia/ausencia de comorbilidad psiquiátrica y las variables de resultado se muestran en la tabla 4 . . Se puede observar que en el grupo de pacientes con presencia de comorbilidad, la media de días de estancia en CT ( $\bar{x}=133)$, el porcentaje de pacientes con alta terapéutica $(34.1 \%)$ y el porcentaje de aquellos que presentan cambios clínicamente relevantes son menores (44.4\%), respecto al grupo con sólo 
trastornos por consumo de sustancias ( $\bar{x}=133,56.5 \%$ y $64.1 \%$, respectivamente). Estos datos se mantienen estables independientemente de los tipos de trastornos presentados por los pacientes: sólo trastornos del Eje I, sólo trastornos del Eje Il o ambos. El análisis que se realiza por trastorno refleja que existen diferencias estadísticamente significativa entre el trastorno del estado de ánimo y la ICA-RT ( $\chi 2=6.622 ; g . I=2 ; p<0.05)$; los trastorno psicóticos, el tipo de alta $(\chi 2=8.244 ; g . l=2 ; p<0.05)$ y el tiempo de estancia $(t=2.019 ; g . l=69.475 ; p<0.05)$; el trastorno límite de la personalidad y el ICA-RT $(\chi 2=15.794 ; g . l .=2 ; p<0.01)$, tipo de alta $(\chi 2=$ $16.2 ; g . l .=2 ; p<0.01)$ y tiempo de estancia $(t=2.763 ; g . l=216 ; p<0.01) y_{1}$ por último, el TDAH y el tiempo de estancia $(t=2.120 ; g . l=216 ; p<0.05)$.

Tabla 4 Análisis comparativo de las variables de resultados entre el grupo con o sin algún trastorno mental además de los trastornos por consumo de sustancias

\begin{tabular}{|c|c|c|c|}
\hline & \multicolumn{3}{|c|}{$\begin{array}{l}\text { PRESENCIA DE ALGÚN TRASTORNO } \\
\text { EJE I Y/O EJE II EN EL ÚLTIMO AÑO }\end{array}$} \\
\hline & $\begin{array}{c}\text { sí } \\
\mathrm{n}(\%)\end{array}$ & $\begin{array}{c}\text { NO } \\
n(\%)\end{array}$ & $p$ \\
\hline $\begin{array}{l}\text { Días estancia CT } \\
\text { (media } \pm \text { DT) }\end{array}$ & $133(80.7)$ & 147 (76.6) & $>0.05$ \\
\hline $\begin{array}{l}\text { Tipo de alta } \\
\text { Alta no terapéutica } \\
\text { Alta por cumplimiento Parcial de } \\
\text { Objetivos } \\
\text { Alta terapéutica }\end{array}$ & $\begin{array}{l}42(33.3) \\
41(32.5) \\
43(34.1)\end{array}$ & $\begin{array}{l}22(23.9) \\
18(19.6) \\
52(56.5)\end{array}$ & $0.004^{* *}$ \\
\hline \multirow[t]{3}{*}{$\begin{array}{l}\text { Impresión clínica al alta sobre la } \\
\text { respuesta al Tratamiento (ICA-RT) } \\
\text { Sin cambios } \\
\text { Cambios minimos } \\
\text { Cambios clinicamente relevantes }\end{array}$} & $\begin{array}{c}39(31) \\
31(24.6) \\
56(44.4)\end{array}$ & $\begin{array}{l}15(16.3) \\
18(19.6) \\
59(64.1)\end{array}$ & $0.010^{*}$ \\
\hline & \multicolumn{3}{|c|}{$\begin{array}{l}\text { PRESENCIA DE ALGÚN TRASTORNO } \\
\text { EE I EN EL ÚLTIMO AÑO }\end{array}$} \\
\hline & $\begin{array}{c}\mathrm{si} \\
\mathrm{n}(\%)\end{array}$ & $\begin{array}{l}\text { NO } \\
\mathrm{n}(\%)\end{array}$ & $P$ \\
\hline $\begin{array}{l}\text { Días estancia CT } \\
\text { (media -DT) }\end{array}$ & $135(83)$ & 141 (76.4) & $>0.05$ \\
\hline $\begin{array}{l}\text { Tipo de alta } \\
\text { Alta no terapéutica } \\
\text { Alta por cumplimiento Parcial de } \\
\text { Objetivos } \\
\text { Alta terapéutica }\end{array}$ & $\begin{array}{c}30(33) \\
32(35.2) \\
29(31.8)\end{array}$ & $\begin{array}{c}34(26.8) \\
27(21.3) \\
66(52)\end{array}$ & $0.009^{* *}$ \\
\hline $\begin{array}{l}\text { Impresión clínica al alta sobre la } \\
\text { respuesta al tratamiento (ICA-RT) } \\
\text { Sin cambios } \\
\text { Cambios minimos } \\
\text { Cambios clínicamente relevantes }\end{array}$ & $\begin{array}{l}28(30.8) \\
28(30.8) \\
35(38.5)\end{array}$ & $\begin{array}{c}26(20.5) \\
21(16.5) \\
80(63)\end{array}$ & $0.001^{* *}$ \\
\hline & \multicolumn{3}{|c|}{$\begin{array}{l}\text { PRESENCIA DE ALGÚN TRASTORNO } \\
\text { EJE II EN EL ÚLTIMO AÑO }\end{array}$} \\
\hline & $\begin{array}{c}\mathrm{si} \\
\mathrm{n}(\%)\end{array}$ & $\begin{array}{l}\text { NO } \\
n(\%)\end{array}$ & $P$ \\
\hline $\begin{array}{l}\text { Días estancia CT } \\
\text { (media } \pm \text { DT) }\end{array}$ & 64 (119.3) & 154 (147.1) & $0.018^{*}$ \\
\hline $\begin{array}{l}\text { Tipo de alta } \\
\text { Alta no terapéutica } \\
\text { Alta por cumplimiento Parcial de } \\
\text { Objetivos } \\
\text { Alta terapéutica }\end{array}$ & $\begin{array}{l}23(35.9) \\
23(35.9) \\
18(28.1)\end{array}$ & $\begin{array}{c}41(26.6) \\
36(23.4) \\
77(50)\end{array}$ & $0.011^{*}$ \\
\hline $\begin{array}{l}\text { Impresión clínica al alta sobre la } \\
\text { respuesta al tratamiento (ICA-RT) } \\
\text { Sin cambios } \\
\text { Cambios minimos } \\
\text { Cambios clinicamente relevantes }\end{array}$ & $\begin{array}{c}18(28.1) \\
18(28.1) \\
28(43.89\end{array}$ & $\begin{array}{l}36(23.4) \\
31(20.1) \\
87(56.5)\end{array}$ & $>0.05$ \\
\hline
\end{tabular}

Nota: los números expresan medias y (desviaciones típicas) para las variables cuantitativas y porcentajes para las variables cualitativas. ${ }^{*} p<0.05 ;{ }^{* *} p<0.01$

\section{Discusión}

Con respecto al tiempo de estancia, se encuentra que la tasa de retención del programa a los tres meses fue moderadamente más elevada que la encontrada en otros trabajos (68.3\%), que oscila en torno al 50\% (Condelli y Dunteman, 1993; De Leon y Schwartz, 1984; Gossop, Marsen, Stewart y Rolfe, 1999; Maglione, Chao y Anglin, 2000; Mulder et al., 2009; Nielsen y Scarpitti, 2002). De acuerdo con otros autores (Condelli y De Leon, 1993; Condelli, 1994; De Leon, 1991), se observa que para que un paciente llegue a presentar mejoría, se requiere al menos tres meses de estancia en $C T$, pues tan sólo un $28 \%$ de los pacientes alcanzan cambios clínicamente relevantes antes de los 90 días. Estos datos son un indicador de que los equipos terapéuticos de las CTs deben diseñar estrategias especificas para aumentar la adherencia al tratamiento y evitar el abandono precoz. Como se ha visto, esta estrategia puede aumentar las mejoras clínicas del paciente durante su estancia en el recurso. Por otro lado, en este estudio también se constata que el porcentaje de personas que salen del programa con un alta terapéutica (91.6\%) obtienen cambios clínicos relevantes en mucha mayor medida que los que se marchan con un alta por cumplimiento parcial de objetivos (29\%).

El otro objetivo del presente estudio fue evaluar la relación entre la presencia de comorbilidad psicopatológica y la retención en tratamiento en la CT. La hipótesis inicial es que aquellos pacientes que presentaran comorbilidad psicopatológica van a permanecer menos tiempo en el recurso y, en definitiva, iban a beneficiarse menos del tratamiento. Los resultados de este estudio han apoyado esta hipótesis, constatándose que la existencia de comorbilidad psicopatológica estuvo asociada a un abandono precoz de CT. Es decir, los pacientes con otros trastornos mentales, además de los relacionados con el consumo de sustancias, permanecen en el recurso menos días, obtienen un menor porcentaje de altas terapéuticas y cambios clínicos menos relevantes. Este hallazgo ha sido también informado por otros autores (Agostini et al., 1991; Broome et al., 1999; Condelli, 1994; Dekel, Benbenishty y Amram, 2004; Levin, Evans, Vosburg, Horton, Brooks y Ng, 2004; Mulder et al., 2009; Nielsen y Scarpitti, 2002; Van Stelle, Blumer y Moberg, 2004).

Los resultados encontrados en este trabajo llevan a cuestionarse por qué aquellas personas que presentan comorbilidad psicopatológica permanecen menos tiempo en las comunidades. $E$, igualmente, es necesario cuestionarse si el modelo de intervención con estos pacientes es el más adecuado, y cuáles son sus posibilidades de mejora.

Las CTs en Andalucía están tradicionalmente enfocadas a una intervención de carácter psicoeducativo, multidisciplinar, dirigida fundamentalmente a la conducta adictiva. Ello conlleva una dificultad a la hora de atender a los pacientes con comorbilidad psicopatológica, en tanto que requiren de otras atenciones especializadas. Por ello, y como se ha comentado con anterioridad, es necesario adaptar estos recursos a personas que presenten comorbilidad psicopatológica (Van Stelle et al., 2004). De este modo, los fundamentos de las tradicionales CTs, como por ejemplo el régimen diario altamente estructura- 
do, fomento de la responsabilidad personal, cambios continuos a través de un proceso gradual o la promoción de valores prosociales, deben mantenerse. Pero es necesario realizar modificaciones que se adapten a las necesidades de los pacientes con síntomas psicopatológicos. Por ejemplo, a) una adaptación del perfil de los profesionales donde se deben incluir expertos de la salud mental con experiencia en el campo de las drogodependencias, b) una reducción de la duración y una mayor flexibilidad en la ejecución de las actividades e intervenciones terapéuticas, c) un mayor énfasis en la orientación e instrucción, d) un menor número de sanciones y confrontaciones, e) una afirmación más explícita de los logros, f) mayor atención a las diferencias individuales $y$, por último, g) una mejor respuesta a las necesidades especiales de desarrollo de los pacientes. Por ejemplo, dado el elevado porcentaje de casos de trastornos de la personalidad y TDAH, deberán diseñarse estrategias de intervención flexibles, lo que cuestionaría el modelo tradicional de sanciones.

La creación de CTs adaptadas a personas con comorbilidad psicopatológicas no es una iniciativa novedosa. Experiencias previas la avalan en cuanto a la eficacia en el tratamiento de estas personas y en cuanto a los beneficios coste-efectivos (De Leon et al., 2000; Sacks, McKendrik, Sacks, Banks y Harle, 2008b; Van Stelle y Moberg, 2004; Van Stelle et al., 2004). En este sentido, distintos señalan que las CTs adaptadas a personas con comorbilidad psicopatológicas deben partir de un modelo integral que se distinga de las CTs tradicionales en dos aspectos fundamentales: mayor flexibilidad y mayor intervención individualizada (De Leon, 1996; Sacks, Banks, McKendrick y Sacks, 2008a; Sacks et al., 2010). Además,

Es importante que los datos de este estudio se interpreten y se extrapolen con la suficiente cautela. En primer lugar, debe tenerse en cuenta las peculiaridades del programa asistencial de las CTs de Andalucía, dado que estos recursos forman parte de una red asistencial coordinada cuyo tiempo de estancia media es de unos seis meses (más reducido que las CTs tradicionales estadounidenses o europeas). En segundo lugar, se optó por considerar una muestra naturalística obtenida en condiciones muy próximas a la práctica clínica diaria con criterios de selección poco restrictivos. Por ello, hay que tener cautela al generalizar las conclusiones a grupos más específicos de pacientes. En este sentido, podría considerarse una limitación el hecho de que se hable de comorbilidad psicopatológica relacionada con el consumo de cocaína, cuando la muestra que estudiada no está compuesta por pacientes con dependencia a cocaína exclusivamente. Siendo esto cierto, no es menos verdad que en caso de haber adoptado esta actitud tan restrictiva en el muestreo, además de problemas de reclutamiento de casos, hubiésemos tenido un grave problema de extrapolación de los resultados, al separarnos excesivamente de la realidad asistencial. En tercer lugar, los instrumentos utilizados para evaluar la evolución de la gravedad de la psicopatología son de carácter autoadministrado, lo que puede suponer una gran limitación en esta población.

Finalmente, cabe decir que una de las potencialidades de este estudio frente a otros es el hecho de que la evaluación de los resultados del tratamiento (ICA-RT) ha sido cumplimentada por los terapeutas y trasformada por los investigadores. Estas diferencias en la medición podrian estar relacionadas con la diversidad de los datos encontrados en otros estudios (Agostini et al., 1991; Alterman et al., 1996; Joe et al., 1995). En futuros estudios, pensamos que se debe utilizar una herramienta exclusiva para valorar, por una parte, las variables de resultados y por otras, variables relacionadas con el tratamiento que hagan conocer qué peso tienen en la eficacia del programa con respecto a las variables del sujeto.

A pesar de estas limitaciones, este es uno de los primeros estudios que se realiza simultáneamente en todas las CTs públicas de Andalucia, por lo que tiene valor epidemiológico y relevancia desde la perspectiva de la planificación de servicios. Los datos aportados ponen de manifiesto que la comorbilidad psicopatológica entre los pacientes con dependencia de cocaína que ingresan en CT es elevada que se asocia a una peor respuesta al tratamiento $y$, lo que es peor, que la probabilidad de abandonar el programa antes de que se den las condiciones para observar mejorías clínicas relevantes es alta. Al mismo tiempo, la menor respuesta entre los pacientes con comorbilidad psicopatológica, nos señala las limitaciones del modelo tradicional de las CTs para abordar la complejidad de esta patología.

\section{Reconocimientos}

Los autores desean reconocer por un lado, la colaboración continúa de todos los trabajadores del área de Atención a las Drogodependencias e Incorporación Social de la Agencia de Servicios Sociales y Dependencia en Sevilla y, por el otro, a los miembros de las seis comunidades terapéuticas públicas de Andalucía que colaboraron de una manera u otra en la realización de estudio. Asimismo, agradecer a la Doctora Marta Torrens y su equipo de Investigación del Parc de Salut Mar (IMIM) de Barcelona, por su apoyo durante el proyecto. Por último, agradecer a todos los participantes del estudio que han hecho posible que este trabajo de la luz.

Este artículo ha sido financiado por el Plan Nacional sobre Drogas (PND 2007i078), el P07.HUM 03089 de la Junta de Andalucia (Proyectos de Excelencia 2007) y el Programa RETICS (Red de Trastornos Adictivos Convocatoria 2012) del Ministerio de Sanidad, Servicios Sociales e Igualdad.

\section{Conflictos de Intereses}

Los autores declaran que no tienen conflicto de intereses para este trabajo

\section{Referencias}

Agostini, V., Nunes, E., Stewart, J. W. y Quitkin, F. M. (1991). Patient factors related to early attrition from an outpatient cocaine 
research clinic: A preliminary report. International Journal of the Addictions, 26, 327-334.

Arenas, F., del Valle, M., López, R., Martín, J. y Tirado, P. (2003). Programa de Intervención. Comunidad Terapéutica en Andalucía. Sevilla: Consejería de Asuntos Sociales. Comisionado para las Drogodependencias. Junta de Andalucía.

Alterman, A. I., Rutherford, M. J. , Cacciola, J. S., McKay, J. R. y Woody, G. E.. (1996). Response to Methadone Maintenance and Counseling in Antisocial Patients with and without Major Depression. Journal of Nervous and Mental Disease, 184, 695-670.

Broome, K. M., Flynn, P. M. y Simpson, D. D. (1999). Psychiatric comorbidity measures as predictors of retention in drug abuse treatment programs. Health Services Research, 34, 791-806.

Bunt, G., Muehlbach, B. y Moed, C. (2008). The therapeutic Community: an international perspective. Substance Abuse, 29, 81-87. doi: 10.1080/08897070802218844.

Condelli, W. S. (1994). Domains of variables for understanding and improving retention in therapeutic communities. International Journal of the Addictions, 29, 593-607.

Condelli, W. S. y Hubbard, R. L. (1994). Relationship between time spent in treatment and client outcomes from therapeutic communities. Journal Substance Abuse Treatment, 11, 25-33.

Condelli, W.S. y Dunteman, G.H. (1993). Issues to consider when predicting retention in therapeutic communities. Journal of Psychoactive Drugs, 25, 239-244.

Condelli, WS. y De Leon, G. (1993). Fixed and Dynamic Predictors of Client Retention in therapeutic communities. Journal Substance Abuse Treatment, 10, 11-16.

Conners, C. K. , Erhardt, D. y Sparrow, E. (1999). Conners adult ADHD rating scales. North Tonawanda, Nueva York: Multi-Health Systems.

Darke, S., Campbell, G. y Popple, G. (2012). Retention, early dropout and treatment completion among therapeutic community admissions. Drug and Alcohol Review, 31, 64-71. doi: 10.1111/j.14653362.2011 .00298

De Leon, G., Sacks, S., Staines, G. y McKendrick, K. (2000). Modified therapeutic community for homeless mentally ill chemical abusers: treatment outcomes. American Journal of Drug and Alcohol Abuse, 26,461-480.

De Leon, G. (1991). Retention in drug-free therapeutic communities. En R.W. Pickens, C.G. Leukefeld y C.R.Schuster (Eds.), Improving drug abuse treatment. NIDA Research Monograph Series, no 106 Rockville, MD: US. Department of Health and Human Services.

Dekel, R., Benbenishty, R., y Amram, Y. (2004). Therapeutic communities for drug addicts: prediction of long-term outcomes. Addictive Behaviors, 29, 833-837.

European Monitoring Centre for Drugs and Drug Addiction (2011). Annual Report 2009: Annual report on the state of the drugs problem in Europe. Recuperado de: http://www.emcdda.europa.eu/ publications/annual-report/2011.

Falck, R.S., Wang, J., Siegal, H.A. y Carlson, R.G. (2004). The prevalence of psychiatric disorder among a community sample of crack cocaine users: an exploratory study with practical implications. Journal of Nervous and Mental Disease , 192, 503-507.

Fernández-Hermida, J. R. , Secades-Villa, R. , Fernández-Ludeña , J. J. y Marina-González, P, A. (2002). Effectiveness of a therapeutic community treatment in Spain: a long-term follow-up study. European Addiction Research, 8, 22-29.

Gossop, M., Marsden, J., Stewart, D., y Rolfe, A. (1999).Treatment retention and 1 year outcomes for residential programmes in England. Drug Alcohol \& Dependence, 57, 89-98.

Greenfield, L., Burgdorf, K., Chen, X., Porowsk, A., Roberts, T., y Herrell, J. (2004). Effectiveness of long-term residential substance abuse treatment for women: findings from three national studies. American Journal of Drug and Alcohol Abuse, 30, 537-550.

Gilchrist, G., Langohr, K., Fonseca, F., Muga, R. y Torrens, M. (2012). Factors associated with discharge against medical advide from and alcohol and drug inpatient detoxification unit in Barcelona between 1993 and 2006. Heroin Addiction and Related Clinical Problems, 14, 35-44.

Hasin, D., Trautman, K., Miele, G., Samet, S., Smith, M., y Endicott, J. (1996). Psychiatric Research Interview for Substance and Mental Disorders (PRISM): Reliability for substance abusers. American Journal of Psychiatry, 153, 1195-1201.

Herrero, M.J., Domingo-Salvany, A., Brugal, M. T., Torrens, M. y Itinere Investigators. (2011). Incidence of psychopathology in a cohort of young heroin and/or cocaine users. Journal of Substance Abuse Treatment, 41, 55-63. doi: 10.1016/j.jsat.2011.01.013

Joe, G.W., Simpson, D.D. y Broome, K. M. (1998). Effects of readiness for drug abuse treatment on client retention and assessment of process. Adicction, 93, 1177-1190.

Levin, F. R., Evans, S. M., Vosburg, S. K., Horton, T., Brooks, y D., Ng, J. (2004). Impact of attention-deficit hyperactivity disorder and other psychopathology on treatment retention among cocaine abusers in a therapeutic community. Addiction Behaviour, 29, 1875-1882.

Lewis, B.J. y Ross, R. (1994). Retention in therapeutic communities: challenges for the nineties. En F. M. Tims., G. De Leon y N. Jainchill (Eds.), Therapeutic community: advances in research and application. NIDA Research Monograph 144.

Maglione, M., Chao, B. y Anglin, D. (2000). Residential treatment of methamphetamine users: correlates of drop-out from the California Alcohol and Drug Data Systems (CADDS), 1994-1997. Addiction Research, 8, 65-79.

Malivert, M., Fatséas, M., Denis, C., Langlois, E. y Auriacombe, M. (2012). Effectiveness of therapeutic communities: a systematic review. European Addiction Research, 18, 1-11. doi: 10.1159/000331007

Messina, N., Wish, E. y Nemes, S. (2000). Predictors of treatment outcomes in men and women admitted to a therapeutic community. American Journal of Drug and Alcohol Abuse, 26,207-227.

Mulder, R., Frampton, C. M., Peka, H., Hampton, G. y Marsters, T. (2009). Predictors of 3 -month retention in a drug treatment therapeutic community. Drug and Alcohol Review, 28, 366-371. doi: 10.1111/j.1465-3362.2009.00050.x 
Nielsen, AL. y Scarpitti, FR. (2002). Predicting Retention in a Therapeutic Community for Incarcerated Substance Abusers Journal of Offender Rehabilitation, 34, 47-65.

Ramos-Quiroga, J. A., Bosch, R., Richarte, V., Velero, S., Gómez-Barros, N., Nogueira, M., Palomar, G., Corrales, M., Sáez-Francàs, N., Corominas, M., Real, A., Vidal, R., Chalita, P. J. y Casas, M. (2012). Validación de criterio y concurrente de la versión española de la Conners Adult ADHD Diagnostic Interview for DSM-IV. Revista de Psiquiatría y Salud Mental, 5, 229-235. doi: 10.1016/j.rpsm.2012.05.004.

Sacks, S., McKendrick, K., Sacks, J. Y. y Cleland, C. M. (2010). Modified therapeutic community for co-occurring disorders: single investigator meta analysis. Substance Abuse, 31, 146-161. doi: 10.1080/08897077.2010.495662

Sacks, S., Banks, S., McKendrick, K., y Sacks, J. Y. (2008a). Modified therapeutic community for co-occurring disorders: a summary of four studies. Journal Substance and Abuse Treament, 34, 112-122.

Sacks, S., McKendrick, K., Sacks, J. Y., Banks, S., y Harle, M. (2008b). Enhanced outpatient treatment for co-occurring disorders: main outcomes. Journal Substance and Abuse Treament, 34, 48-60.

Secades-Villa, R. y Fernández-Hermida, J. R. (2000). Cómo mejorar las tasas de retención en los tratamientos de drogodependenicas. Adicciones, 12, 353-363.

Secades-Villa, R. y Benavente, M. (2000). Predictores de la retención en una Comunidad Terapéutica para Drogodependientes. Adicciones, 12, 365-371.

Torrens, M., Serrano, D., Astals, M., Perez-Dominguez, G., y MartinSantos, R. (2004). Diagnosing comorbid psychiatric disorders in substance abusers: validity of the Spanish versions of the Psychiatric Research Interview for Substance and Mental Disorders and the Structured Clinical Interview for DSM-IV. American Journal of Psychiatry, 161, 1231-1237.

Tull, M. T. y Gratz, K. L. (2012). The impact of borderline personality disorder on residential substance abuse treatment dropout among men. Drug Alcohol \& Dependence, 1, 121, 97-102. doi: 10.1016/j. drugalcdep.2011.08.014

Van Stelle, K. R., Blumer, C. y Moberg, D. P. (2004).Treatment retention of dually diagnosed offenders in an institutional therapeutic community. Behavioral Sciences \& The Law, 22, 585-97.

Vergara-Moragues, E., González-Saiz, F., Lozano Rojas, O. M., Betanzos Espinosa, P., Fernández Calderón, F., Bilbao Acedos, l., Pérez Garcia, M., Verdejo-Garcia, A. (2012). Psychiatric Comorbidity in cocaine users treated in therapeutic community: Substance-induced versus independent disorders. Psychiatry Research, 200, 734-41. doi: 10.1016/j.psychres.2012.07.043

Vergara-Moragues, E. (2010). Comorbilidad psicopatológica en pacientes con dependencia de cocaina tratados en comunidad terapéutica. Tesis Doctoral en Psicología. Granada: Facultad de Psicología. Universidad de Granada.

Wise, B. K., Cuffe, S. P. y Fischer, T. (2001). Dual diagnosis and successful participation of adolescents in substance abuse treatment. Journal of Substance Abuse Treatment, 21, 161-165. 